\title{
Lung simulation to support non-invasive pulmonary blood flow measurement in Acute respiratory distress syndrome animals*
}

\author{
Minh C. Tran, Douglas C. Crockett, Arun Joseph, Federico Formenti, Phi A. Phan, Stephen J. Payne, \\ Andrew D. Farmery
}

\begin{abstract}
Patients undergoing mechanical lung ventilation are at risk of lung injury. A noninvasive bedside lung monitor may benefit these patients. The Inspired Sinewave Test can measure cardio-pulmonary parameters noninvasively. A lung simulation is proposed to improve the pulmonary blood flow measurement results in this test. Results have shown an improvement in the measurement of blood flow with simulated data. The new method was applied to analyse 12 pigs' data before lung injury (control) and after lung injury (ARDS model). Paired blood flow measurements were performed by both the IST (noninvasive) and thermodilution (invasive). In the control group, the bias of the two methods was negligible $(0.02 \mathrm{~L} / \mathrm{min})$, and the limit of agreement was from -1.20 to $1.18 \mathrm{~L} / \mathrm{min}$. The bias was $-0.68 \mathrm{~L} / \mathrm{min}$ in the ARDS group and with a broader limit of agreement $(-2.49$ to $1.13 \mathrm{~L} / \mathrm{min})$.
\end{abstract}

Clinical Relevance - the inspired sinewave test can be used to measure absolute cardiac output noninvasively in mechanically ventilated subjects with and without acute respiratory distress syndrome.

\section{INTRODUCTION}

Acute respiratory distress syndrome (ARDS) and acute lung injury are forms of respiratory failure caused by widespread rapid inflammation in the lungs. ARDS is usually treated with mechanical ventilation in Intensive Care Units (ICU). In 2019, a global pandemic occurred with the spreading of the Coronavirus disease 2019 (COVID-19), related closely to the ARDS [1]-[3]. Although ARDS necessitates mechanical ventilation, the latter can itself worsen the underlying lung injury. Therefore optimal ventilator setting and continuous bedside lung monitoring can benefit patients with ARDS.

Several bedside lung monitor methods have been developed and validated [4], [5]. According to the lung physiological parameters monitored at the bedside, clinicians can benefit patients by providing optimal/least injurious ventilator settings. The inspired sinewave test (IST) is a noninvasive method to quantify lung function parameters, including lung heterogeneity, effective lung volume and pulmonary blood flow [6]-[8]. The IST does not use ionising radiation and does not require patient effort.

The monitoring of the pulmonary blood flow or cardiac output can guide surgical patients at high risk of haemorrhage

*Research supported by NIHR and MRC research grants. M.C.T. and S.J.P. are with the Institute of Biomedical Engineering, University of Oxford, Oxford, UK. (Corresponding author, e-mail: minh.tran@chch.ox.ac.uk).

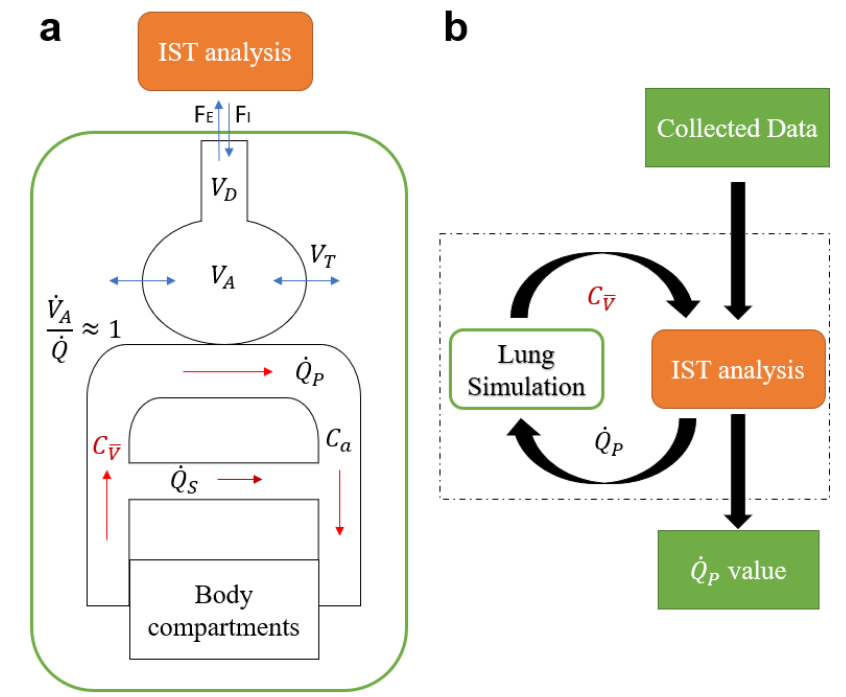

Fig. 1. Schematic diagram of the lung simulation (panel a) and the model analysis program using the lung simulation (panel b).

or haemodynamic dysfunction [9]. The current gold standard for measuring cardiac output is thermodilution [10]. Because the thermodilution procedure requires an invasive setup, the use of this procedure has been declining in favour of less invasive methods. The IST can measure the cardiac output via a respiratory gas exchanged technique.

One advantage of the IST in measuring cardiac output is that theoretically, it can be recovered without measuring the mixed venous concentration of tracer gas, thus allowing it to be completely noninvasive. Early studies, however, showed that recovery of cardiac output was imprecise at higher values.

We hypothesised that we could achieve more accurate pulmonary blood flow results by including a simulation of the mixed venous signal in our analysis. We test this hypothesis in simulated experimental (porcine) datasets. The relationship between pulmonary blood flow and PEEP level was assessed in both control and injured lungs (or ARDS animal model).

F.F. is with the Centre for Human \& Applied Physiological Sciences, King's College London, London, UK.

D.C.C., A.J., P.A.P. and A.D.F. are with the Nuffield Department of Clinical Neuroscience, University of Oxford, Oxford, UK. 


\section{MATERIAL AND METHOD}

\section{A. Experimental protocol}

Twelve anaesthetised pigs with $29 \mathrm{~kg}$ (SD 2) were studied by the IST both before lung injury (control) and after lung injury (ARDS). Lachmann's method was applied to induce lung injury to simulate the ARDS in an animal model. The experiments were performed in the Uppsala University Hospital. Each animal was studied on the same day from 9 am to $7 \mathrm{pm}$. Animal preparations were mentioned elsewhere [5]. The experiments followed the Report of in vivo experiments (ARRIVE). Characteristics of animals are included in Table 1.

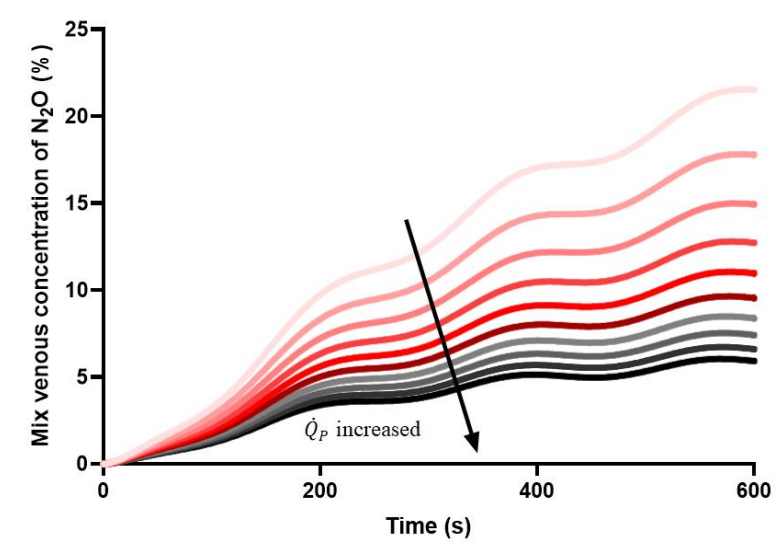

Fig. 2. Simulation of the mixed venous concentration of the $\mathrm{N}_{2} \mathrm{O}$ with increased pulmonary blood flow $\left(\dot{Q}_{P}\right)$

During the preparation, the mechanical ventilator was set to deliver in volume-controlled ventilation mode with 20-25 breaths $/ \mathrm{min}$ respiration rate and tidal volume of $10 \mathrm{ml} / \mathrm{kg}$. For each animal, measurements were repeated twice by the IST and thermodilution at each different positive end-expiratory pressure (PEEP) level. While other parameters of the ventilator setting were kept unchanged, the PEEP levels were incrementally increased from 0 to $5,10,15$ and $20 \mathrm{cmH}_{2} \mathrm{O}$. Changes in PEEP level typically produce changes in resting lung volume capacity and a reduction in pulmonary blood flow. After measurements in the healthy animals, lung injury was induced, and the exact measurements were repeated. For injured lungs, the PEEP level was increased from 5 to 10,15 and $20 \mathrm{cmH}_{2} \mathrm{O}$.

\section{B. Inspired Sinewave Test}

IST is a simple and noninvasive method to measure cardiopulmonary indices by using oscillating gas. [11], [12]. By using oscillating tracer gas, the dead space volume (VD), effective lung volume (ELV) and blood flow rate $\left(\dot{Q}_{P}\right)$ can be calculated. IST applies a forced oscillation of a low dose $\mathrm{N}_{2} \mathrm{O}$ tracer gas with a sinusoidal period which can be determined by the user.

The IST analysis program takes the input (concentration of $\mathrm{N}_{2} \mathrm{O}$ in both inhaled $-F_{I}$ and exhaled breaths $-F_{E}$ ) and produces the outputs (VD, ELV and $\dot{Q}_{P}$ ). A one-compartment lung was assumed to represent the whole lung in the breathby-breath analysis. The mass balance equation of the tracer gas of two consecutive breath $(n-1$ and $n)$ is:
$F_{E, n-1} V_{A}+F_{\mathrm{I}, n}\left(V_{T, n}-V_{D}\right)+F_{E, n-1} V_{D}-\lambda \times \dot{Q}_{P}$

$\times\left(F_{E, n}-C_{\bar{v}}\right) \Delta t_{n}=V_{A} F_{E, n}+V_{T, n} F_{E, n}$

where:

$F_{I, n}:$ the inspired concentration of breath $\mathrm{n}^{\text {th }}$.

$F_{E, n}$ : the end expired concentrations of breath $n$.

$\lambda$ : the solubility of $\mathrm{N}_{2} \mathrm{O}$ in blood, $\lambda=0.47$.

$C_{\bar{v}}$ : the mixed venous concentration,

$\Delta t_{n}$ : the duration of breath $\mathrm{n}^{\text {th }}$.

$V_{T, n}$ : the tidal volume of breath $\mathrm{n}^{\text {th }}$.

In previous studies, the unknown mixed venous concentration $\left(C_{\bar{v}}\right)$ was assumed to equal to the mean of the inspired sinewave concentration at a steady-state $F_{I}^{0}$. This assumption was based on the notion that the mean mixed venous, inspired and end-tidal concentrations would all be equal in equilibrium. It was also assumed that the oscillation would be fully damped in the mixed-venous signal. However, due to increased $\dot{Q}_{P}$ errors at high flows, we suspected that this assumption might not be valid. We, therefore, propose a method to simulate the mixed venous concentration from a developed lung simulation to support the calculation of the absolute $\dot{Q}_{P}$ value.

TABLE I. CHARACTERISTIC OF THE ANIMALS (N=12) [MEAN (SD)]. PVALUES SHOW RESULTS OF EITHER PAIRED STUDENT'S T-TEST (PARAMETRIC DATA). $\mathrm{CO}=$ CARDIAC OUTPUT CALCULATED BY THERMODILUTION, $\mathrm{PAO}_{2}=$ ARTERIAL $\mathrm{O}_{2}$ PARTIAL PRESSURE, FIO ${ }_{2}=$ FRACTION OF INSPIRED $\mathrm{O}_{2}, \mathrm{PFR}=$ $\mathrm{PAO}_{2} / \mathrm{FIO}_{2}$ RATIO.

\begin{tabular}{l|c|c|r}
\hline Parameter & Control & ARDS & p \\
\hline Weight $(\mathrm{Kg})$ & $29(2)$ & - & - \\
$\mathrm{HR}(\mathrm{bpm})$ & $86(12)$ & $85(11)$ & 0.42 \\
$\mathrm{CO}(\mathrm{L} / \mathrm{min})$ & $3.2(0.4)$ & $3.5(0.8)$ & 0.26 \\
$\mathrm{pH}$ & $7.38(0.07)$ & $7.25(0.08)$ & 0.0004 \\
$\mathrm{FIO}_{2}(\%)$ & $0.4(0.1)$ & $0.8(0.1)$ & 0.0002 \\
$\mathrm{PaO}_{2}(\mathrm{mmHg})$ & $144(30)$ & $96(28)$ & 0.39 \\
\hline
\end{tabular}

\section{Lung simulation to support IST analysis}

The tidal lung simulation was developed to support the IST analysis program. The schematic diagram was described in Fig.1, panel a. The lung was assumed to be homogeneous and was represented by one compartment only. This lung simulation was introduced and validated elsewhere [6], [13].

In this work, the lung simulation was used to generate the mixed-venous concentration of $\mathrm{N}_{2} \mathrm{O}\left(C_{\bar{V}}\right)$ for the IST analysis. However, the mixed-venous concentration was influenced by the pulmonary blood flow $\dot{Q}_{P}$. Fig. 2 shows mixed-venous $\mathrm{N} 2 \mathrm{O}$ concentration vs blood flow changes while performing the IST in simulated data. Therefore, a simple optimisation was integrated into the algorithm to suggest the $C_{\bar{V}}$ values before calculating the $\dot{Q}_{P}$. A simple diagram of this method was shown in Fig.1 panel b.

Parameters for the lung simulation were taken from the literature [14]. These data represented a healthy lung with different pulmonary blood flow values, ranging from $2 \mathrm{~L} / \mathrm{min}$ to $7 \mathrm{~L} / \mathrm{min}$. The lung model simulated a $70 \mathrm{Kg}$ man with 2.5 
$\mathrm{L}$ of alveolar volume and $150 \mathrm{~mL}$ of deadspace volume. The lung was assumed to be homogeneous. The computational model was developed in Matlab-Simulink (www.mathworks.com) with the ode45 solver. Sensitivity analysis for the model and the model verification was performed elsewhere [6], [13], [15].

\section{Statistical analysis}

Linear regression and Bland-Altman are applied to analyse the relationship and correlation between pair measurements of thermodilution and the IST [16].
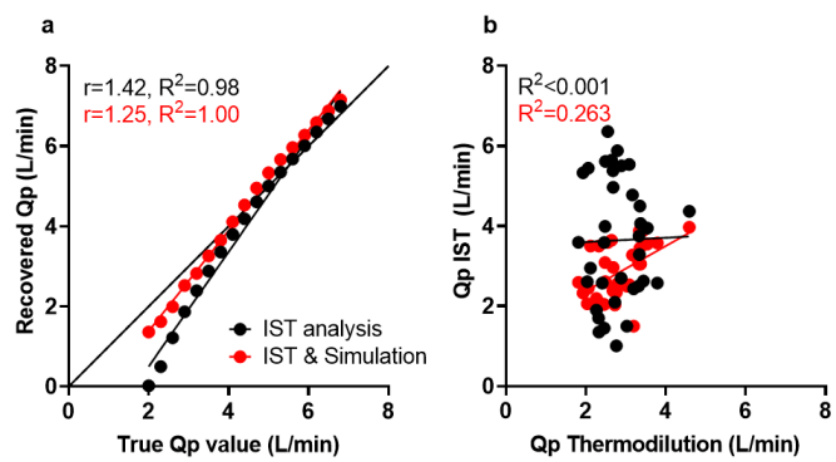

Fig. 3. Comparison of pulmonary blood flow measurement by the conventional IST analysis vs IST analysis using lung simulation. Panel a shows the comparison in simulated data with $20 \%$ tidal volume noise. Panel b shows the comparison of both methods (paired IST and thermodilution) in control pigs.

\section{RESULTS}

A total of 130 paired measurements in twelve animals at different PEEP levels were recorded. The values of repeated measurements of the pulmonary blood flow were averaged due to the negligible variations (5\% in thermodilution and $10 \%$ in IST).

Fig. 3 shows improvements of the new method compared to the conventional IST analysis in simulated data (panel a). The $\mathrm{R}^{2}$ was increased, and the slope approximated to 1 . Furthermore, the new method also corrected the underestimation of results at low $\dot{Q}_{P}$ (less than $3 \mathrm{~L} / \mathrm{min}$ ). In panel b, the $\dot{Q}_{P}$ measured by the IST analysis with lung simulation (red points and line) showed an improved correlation with the value measured by the thermodilution in healthy pigs $\left(\mathrm{R}^{2}=0.263\right)$.

Fig. 4 shows the paired comparison of the absolute $\dot{Q}_{P}$ measured by IST with simulation support vs thermodilution in 12 pigs. Generally, the correlation between the two measurements was more robust in the control group than in the ARDS group. Average pulmonary blood flow measured by thermodilution was $2.8( \pm 0.6) \mathrm{L} / \mathrm{min}$ in control and 3.0 $( \pm 0.6) \mathrm{L} / \mathrm{min}$ in ARDS. These values of IST were $2.9( \pm 0.6)$ $\mathrm{L} / \mathrm{min}$ in control and $3.7( \pm 1.2) \mathrm{L} / \mathrm{min}$. As the PEEP was increased, the pulmonary blood flow slightly decreased by 0.9 $\mathrm{L} / \mathrm{min}$ overall. Furthermore, at the PEEP 5 and $10 \mathrm{cmH}_{2} \mathrm{O}$ in the ARDS group, IST overestimated the blood flow compared to thermodilution by $30 \%$.

In the control group, the standard deviation at each PEEP level was smaller than the same results in the ARDS group.
In panel c, the bias was about $0.02 \mathrm{~L} / \mathrm{min}$, and the limit of agreement was from -1.2 to $1.18 \mathrm{~L} / \mathrm{min}$. In panel d, the ARDS group had a more considerable bias than the control group (0.68L/min). In Fig.4 panel d, the bias showed an incensement when the absolute value is raised.
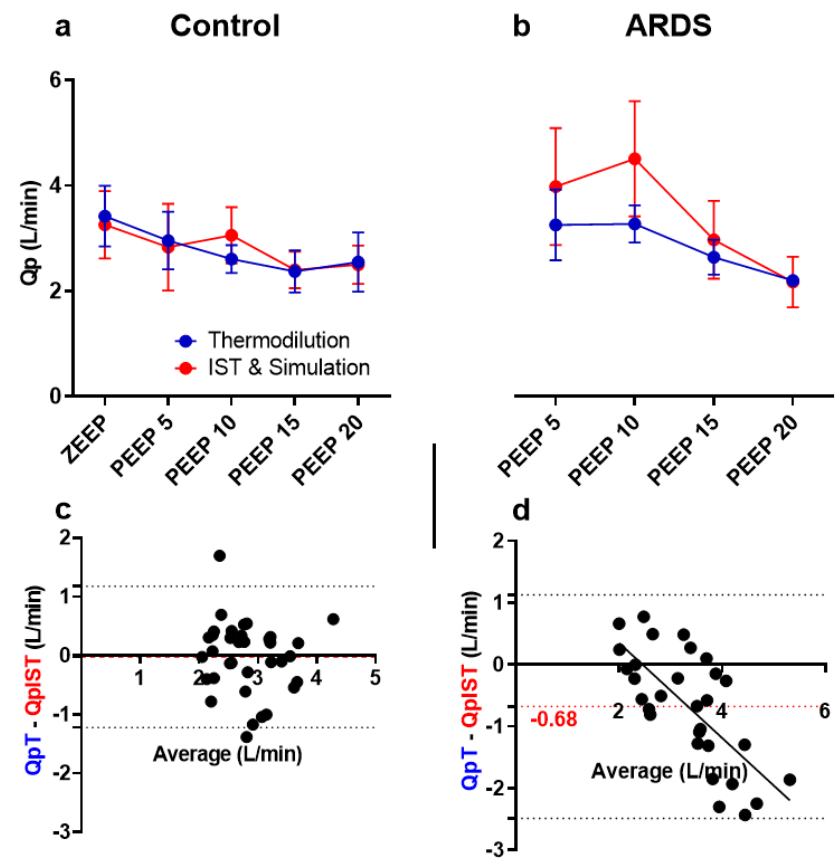

Fig. 4. Comparison of pulmonary blood flow measured by the thermodilution and IST in both control and ARDS lungs. Panel $a$ and $b$ show the effect of PEEP levels on pulmonary blood flow. Mean and standard deviation are shown. Panel $\mathrm{c}$ and $\mathrm{d}$ are Bland-Altman of the comparison between measurements by IST and Thermodilution. Red dash lines are the mean bias, and black dash lines are the limit of agreement.

\section{DISCUSSION}

The results have shown that in both uninjured lungs and injured lungs (ARDS model), IST can measure pulmonary blood flow accurately in mechanically ventilated subjects. This study applied a new lung simulation to support blood flow measurement in the IST to overcome a previous limitation. These results improve the impact of the IST at bedside lung monitoring, which can be used to suggest the optimal mechanical ventilation setting.

Thermodilution is widely applied and considered the gold standard method to monitor cardiac output. However, it is not used widely as it is highly invasive and is complicated to set up [17]. Clinicians prefer noninvasive and less skill demanding methods such as a Doppler ultrasound for pulmonary blood flow measurement. Respiratory measurements like the IST can potentially help with idealised and objective pulmonary blood flow monitoring. The BlandAltman analysis of absolute values showed a good agreement in between IST and thermodilution $(0.02 \mathrm{~L} / \mathrm{min}$ in control and $-0.68 \mathrm{~L} / \mathrm{min}$ in ARDS models). The Bland-Altman plot in control group illustrated a normal distribution, while in the ARDS group, the error increased with the absolute value. Therefore, there is room to develop a statistical method to 
correct the IST measurement.

The IST has been shown to be a suitable bedside lung monitor. The inspired sinewave technique offers several strengths, including accurate pulmonary parameters measurements, commercial availability and its ease of use at the bedside [5], [6], [13]. In addition, as IST does not rely on a fixed inspiratory flow, it can be used spontaneously in ventilating patients.

Both thermodilution and the IST captured the decline of pulmonary blood flow when the PEEP level increased. According to Fig. $4 \mathrm{a}$ and b, when the PEEP levels increased, the cardiac output slightly declined in both control and ARDS groups. PEEP optimisation might reduce the development of lung induced injury and ARDS [18], but there is a trade-off with reducing cardiac output. Further research should be carried on to understand the relationship between the IST parameters and the optimal setting of the mechanical ventilators.

This work contains several limitations. The trending of the cardiac output measured by the IST was not compared in this research. Due to the experimental setup, cardiac output was kept unchanged, except for the changes caused by changes in PEEP. Therefore this study only compared absolute value measurements by pairing IST and thermodilution. Other limitations of this study are that the use of a pig model does not translate directly to humans, and the use of saline-lavage does not entirely comprise all the features of ARDS lungs.

In another research, a proof-of-concept study in eight different pigs concluded that IST and thermodilution have good agreement in capturing the trend when the cardiac output changed [7]. Our study fitted into the gap of the absolute value comparison. However, there is still room for developing the blood flow measurement by the IST in the future. Future development of IST should include assessing its use in mechanically ventilated human patients and patients in the Intensive Care Unit (ICU). A pilot study has been established to test in the John Radcliff Hospital, Oxford.

\section{CONCLUSION}

The mathematical simulation was introduced to boost the performance of the noninvasive beside lung monitor test (IST). We conclude that the IST is a suitable test for mechanically ventilated animals. Furthermore, the IST can measure accurate pulmonary blood flow with small biases than thermodilution in healthy and ARDS model lungs. The results of this work support further development of the IST and its translation to human medicine.

\section{REFERENCES}

[1] J. Phua et al., "Intensive care management of coronavirus disease 2019 (COVID-19): challenges and recommendations," Lancet Respir. Med., vol. 2019, no. 20, pp. 1-12, 2020, doi: 10.1016/s2213-2600(20)30161-2.

[2] C. Wu et al., "Risk Factors Associated with Acute Respiratory Distress Syndrome and Death in Patients with Coronavirus Disease 2019 Pneumonia in Wuhan, China," JAMA Intern. Med., vol. 180, no. 7, pp. 934-943, 2020.

[3] D. Chiumello et al., "Physiological and quantitative CT-scan characterization of COVID-19 and typical ARDS: a matched cohort study," Intensive Care Med., vol. 46, no. 12, pp. 21872196, 2020, doi: 10.1007/s00134-020-06281-2.

[4] M. C. Tran et al., "Bedside monitoring of lung volume available for gas exchange," Intensive Care Med. Exp., vol. 9, no. 1, 2021, doi: 10.1186/s40635-020-00364-6.

[5] D. C. Crockett et al., "Validating the inspired sinewave technique to measure the volume of the 'baby lung' in a porcine lung-injury model," Br. J. Anaesth., vol. 124, no. 3, pp. 345-353, 2020, doi: 10.1016/j.bja.2019.11.030.

[6] M. C. Tran et al., "A tidal lung simulation to quantify lung heterogeneity with the Inspired Sinewave Test," in 2020 42nd IEEE (EMBC), 2020, pp. 2438-2441, doi: 10.1109/EMBC44109.2020.9176375.

[7] R. Bruce et al., "Noninvasive cardiac output monitoring in a porcine model using the inspired sinewave technique: a proofof-concept study," Br. J. Anaesth., vol. 123, no. 2, pp. 126134, 2019, doi: 10.1016/j.bja.2019.02.025.

[8] R. M. Bruce et al., "Assessment of ventilatory heterogeneity in chronic obstructive pulmonary disease using the inspired sinewave test," Int. J. COPD, vol. 16, pp. 401-413, 2021, doi: 10.2147/COPD.S264360.

[9] H. D. Aya et al., "Goal-directed therapy in cardiac surgery: A systematic review and meta-analysis," Br. J. Anaesth., vol. 110, no. 4, pp. 510-517, 2013, doi: 10.1093/bja/aet020.

[10] D. A. Reuter et al., "Cardiac output monitoring using indicator-dilution techniques: Basics, limits, and perspectives," Anesth. Analg., vol. 110, no. 3, pp. 799-811, 2010, doi: 10.1213/ANE.0b013e3181cc885a.

[11] C. E. Hahn et al., "Gas exchange in a three-compartment lung model analyzed by forcing sinusoids of N2O.," J. Appl. Physiol., vol. 75, no. 4, pp. 1863-1876, 1993.

[12] C. E. W. Hahn, "Oxygen respiratory gas analysis by sine-wave measurement: a theoretical model.," J. Appl. Physiol., vol. 81, no. 2, pp. 985-997, 1996, doi: 10.1152/jappl.1996.81.2.985.

[13] M. C. Tran et al., "Lung heterogeneity and deadspace volume in acute respiratory distress syndrome animals using the inspired sinewave test," J. Physiol. Meas., vol. 0, no. 0, pp. 110, 2020, doi: 10.1080/07391102.2020.1758788.

[14] J. S. Yem, et al., "A tidally breathing model of ventilation, perfusion and volume in normal and diseased lungs," Br. J. Anaesth., vol. 97, no. 5, pp. 718-731, 2006, doi: 10.1093/bja/ael216.

[15] M. C. Tran et al., "Simulation - based optimisation to quantify heterogeneity of specific ventilation and perfusion in the lung by the Inspired Sinewave Test," Sci. Rep., no. 0123456789, pp. 1-10, 2021, doi: 10.1038/s41598-021-92062-w.

[16] J. M. Bland and D. G. Altman, "Agreement between methods of measurement with multiple observations per individual," $J$. Biopharm. Stat., vol. 17, no. 4, pp. 571-582, 2007, doi: $10.1080 / 10543400701329422$.

[17] P. E. Marik, "Noninvasive cardiac output monitors: A state-of the-art review," J. Cardiothorac. Vasc. Anesth., vol. 27, no. 1, pp. 121-134, 2013, doi: 10.1053/j.jvca.2012.03.022.

[18] L. Gattinoni et al., "Positive end-expiratory pressure: How to set it at the individual level," Ann. Transl. Med., vol. 5, no. 14, pp. 1-10, 2017, doi: 10.21037/atm.2017.06.64. 